\title{
Survie cellulaire : différences et différenciation
}

> Les mécanismes de la régulation de la survie Pierre H. Vachon cellulaire et de l'apoptose sont d'une nature très complexe, impliquant de nombreux intervenants et de nombreuses voies de signalisation aussi bien dans la prise de décision de survivre (ou de mourir) que dans l'exécution de l'apoptose proprement dite. II en va de même pour l'anoïkose, une forme d'apoptose régulée principalement par les interactions cellule-matrice extracellulaire médiées par les intégrines. Or, la survie cellulaire, l'apoptose et l'anoïkose comportent des différences mécanistiques supplémentaires dépendant des tissus et des types cellulaires spécifiquement concernés. Incidemment, des études de la survie cellulaire effectuées dans un tissu particulier, l'épithélium intestinal humain, ont mis au jour un degré additionnel de complexité dans la régulation de ces processus: l'activation de mécanismes distincts selon l'état de différenciation cellulaire. <

L'apoptose est un processus finement régulé qui exerce des rôles cruciaux dans le développement, le maintien et le renouvellement tissulaire [1]. On sait que l'apoptose doit être maintenue en état de suppression lorsqu'elle n'est pas requise: de ce fait, toute cellule requiert des signaux de survie. L'apoptose peut également être induite par certaines cytokines pro-inflammatoires, ainsi que par diverses agressions causées par les radicaux libres, les toxines, les radiations, les agents susceptibles d'endommager l'ADN, etc. [1, 2]. Dans tous les cas, les spasmes mortels typiques d'une cellule apoptotique incluent un bourgeonnement (blebbing) membranaire, une condensation nucléaire, une dégradation d'ADN et d'organites, ainsi qu'un rétrécissement cellulaire qui aboutit à la formation d'un corpuscule apoptotique éliminé par phagocytose ou évacué dans une lumière.

\section{Survie cellulaire et voie commune de l'apoptose}

Les homologues $\mathrm{Bcl}-2$ constituent un centre décisionel critique de l'apoptose, agissant en tant que



suppresseurs (antiapoptotiques à multidomaines [ $\mathrm{BH} \mathrm{l}$ à $\mathrm{BH} 4]$, soit $\mathrm{BCl}-2, \mathrm{Bcl}-\mathrm{X}_{\mathrm{L}}, \mathrm{Mcl}-$ 1 , etc.), activateurs (pro-apoptotiques à multidomaines [BHl à BH3], soit Bax, Bak, Bok) et sensibilisateurs (pro-apoptotiques à domaine $\mathrm{BH} 3$ seulement, soit Bad, Bid, Bim, Bmf, etc.) [2, 3]. De façon générale, les suppresseurs interagissent avec les activateurs et les empêchent de former des pores dans les mitochondries. De leur côté, les sensibilisateurs cherchent principalement à lier les suppresseurs pour les inhiber, donnant ainsi libre cours aux activateurs; certains sensibilisateurs interagissent également avec des activateurs de façon à potentialiser leurs fonctions $[2,3]$ (Figure 1). La perte d'intégrité de la membrane mitochondriale, causée principalement par les activateurs, mène notamment à la libération du cytochrome $c$ dans le cytosol. Celui-ci agit en tant que cofacteur, avec APAF-1 (apoptosis protease activating factor-1), pour recruter la forme inactive de la caspase initiatrice CASP-9, et former ainsi l'apoptosome conduisant à son activation [2, 3] (Figure 1). À son tour, CASP-9 amorce de manière irréversible une cascade amplificatrice d'activations de caspases effectrices, comme CASP-3 et CASP-7, qui iront cliver leur nombreux substrats (actine, kinases, lamines, etc.). De plus, certains

Article reçu le 2 juillet 2005, accepté le 4 novembre 2005. 
effecteurs apoptotiques seront plutôt activés par les caspases effectrices, comme l'homologue sensibilisateur Bid et la CAD (caspase-activated DNAse) [2-4] (Figure 1). Ainsi, la régulation de l'apoptose dépend largement d'un équilibre entre les activités anti- et pro-apoptotiques de plusieurs homologues $\mathrm{Bcl}-2$ [2, 3]. Un tel équilibre s'établit en tout premier lieu via une modulation de l'expression des divers homologues, selon le stimulus de survie et le type cellulaire concerné. Des modulations post-transcriptionnelles ou post-traductionnelles (phosphorylation, séquestration, etc.) viennent également contribuer à cet équili-

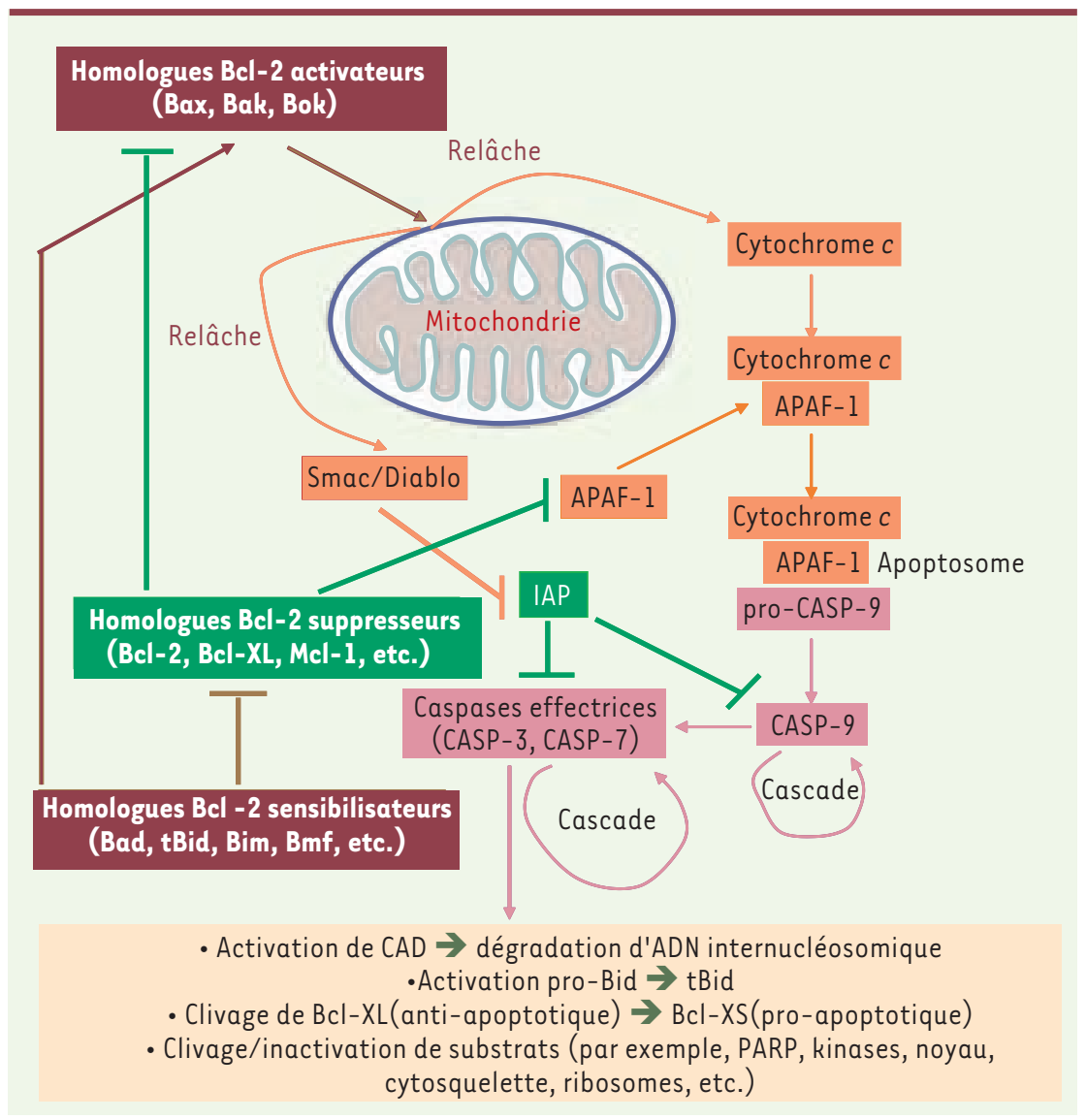

Figure 1. Voie commune de régulation de l'apoptose. À titre de centre décisionnel d'entrée en apoptose, les homologues $\mathrm{Bcl}-2$ influencent notamment l'intégrité des mitochondries et régissent ainsi la formation de l'apoptosome. Les homologues suppresseurs (anti-apoptotiques) d'apoptose peuvent lier et séquestrer APAF-1, et inhiber les homologues activateurs (pro-apoptotiques) susceptibles de provoquer l'ouverture de pores dans la membrane mitochondriale: cette ouverture, quand elle intervient, entraîne la libération du cytochrome $c$ et des inhibiteurs d'IAP dans le cytosol. D'autres homologues pro-apoptotiques, dits sensibilisateurs, ont pour fonction d'inhiber les homologues anti-apoptotiques; certains d'entre eux interagissent également avec des activateurs pour potentialiser les fonctions de ces derniers. Le facteur APAF-1 et le cytochrome $c$ peuvent alors coopérer pour dimériser la pro-CASP-9, formant ainsi l'apoptosome et la mettant aussitôt en activation. II s'ensuivra une cascade amplificatrice d'activation de caspases effectrices. APAF- 1 : apoptosis protease activating factor- 1 ; CAD : caspase-activated internucleosomal DNAse; IAP : inhibitors of apoptosis proteases; PARP : poly(ADP-ribose) polymerase; pro-CASP-9 : précurseur inactif de la caspase initiatrice CASP-9.
À l'instar des facteurs de croissance, hormones et cytokines, les interactions cellule-matrice extracellulaire (MEC) jouent un rôle majeur dans la régulation des divers processus cellulaires, dont le maintien de la survie [3, 5-13]. De ce fait, toute perturbation dans les interactions cellule-MEC induit une forme spécifique d'apoptose : l'anoïkose [3, 5, 10-13]. Les fonctions biologiques attribuées aux interactions cellule-MEC sont principalement assurées par les récepteurs de type intégrine $(\alpha \beta)$ [3, 5-12] : si la liaison d'une intégrine à son ligand crée un lien physique avec le cytosquelette, elle déclenche également une gamme étendue de signaux de transduction [5-9]. Ce sont les intégrines ayant la sous-unité $\beta 1$ en commun qui constituent la majorité des récepteurs pour les constituants de la MEC [5-9]. L'intégrine $\alpha 6 \beta 4$, un récepteur des laminines, est quant à elle exprimée exclusivement dans les épithéliums [5-9].

Bien que relativement avancée, la compréhension des bases moléculaires exactes de la signalisation assurée par les intégrines $\beta 1$ demeure incomplète $[3,6-12]$. On sait désormais que certaines voies comme celles de la phosphatidylinositol 3-kinase (PI3K)/Akt, de MEK (mitogen-activated protein kinase (MAP) kinase)/Erk (extracellular regulated kinase) ou de SAPK (stress-activated MAP kinases), telles les protéines JNK (c-Jun $\mathrm{N}$-terminal kinases) et $\mathrm{p38}$, peuvent être activées seules ou en combinaison [3, 6-11]. De plus, la signalisation par les intégrines $\beta 1$ repose en bonne partie sur Fak (focal adhesion kinase) $[6-8,14,15]$ (Figure 2). Cette diversité de signalisation serait largement attribuable à la formation de cassettes de signalisation distinctes via 
le recrutement par Fak d'un répertoire croissant de partenaires, notamment échangeurs, adapteurs, échafaudeurs, petites GTPases et autres kinases (Src, Ras, Shc, SoS, etc.) [6-8, 14, 15] (Figure 2). La signalisation de type intégrine peut également s'implanter grâce à certaines sous-unités intégrines $\alpha$, une association avec des protéines membranaires (comme les cavéolines) ou d'autres kinases, comme ILK (integrin-linked kinase) [3, 6-8, 10-12, 15] (Figure 2). Enfin, l'intégrine $\alpha 6 \beta 4$ participe également à la stimulation des voies PI3-K/Akt ou MEK/Erk selon le type épithélial étudié $[7,8,15]$. II est maintenant bien établi qu'un répertoire donné d'intégrines exprimées permet non seulement de déclencher des signaux spécifiques à un type cellulaire précis, mais également une modulation différentielle des divers processus cellulaires au sein d'un même tissu $[5-9,15]$.

\section{Suppression et induction de l'anoïkose}

Tout comme l'apoptose, l'anoïkose joue une série de rôles importants au cours de l'embryogenèse et de l'organogenèse, ainsi que dans le maintien et le renouvellement des tissus [3, 6, 10-12]. Étant donné que la grande majorité des cellules requièrent un ancrage sain à leur MEC pour survivre, on peut dire des interactions cellule-MEC qu'elles suppriment l'anoïkose [3, 6, 10-13, 15]. Les mécanismes moléculaires précis qui régissent la suppression de l'anoïkose res-

Figure 2. Signalisation intégrines $\beta 1$-Fak, survie cellulaire et suppression de l'anoïkose. La liaison d'une intégrine $(\alpha) \beta 1$ à son ligand de la matrice extracellulaire (MEC) permet principalement le recrutement et l'activation de Fak. D'une part, Fak active la PI3-K directement, ou indirectement via la tyrosine kinase Src ou une IRS, menant à l'activation d'Akt. Akt peut être également activé via l'ILK. Akt activé phosphoryle les homologues Bcl-2 pro-apoptotiques Bad et Bax pour les inhiber, en plus de phosphoryler la pro-CASP-9 pour renforcer son état inactif. Akt phosphoryle également la kinase pro-apoptotique ASK-1, afin de l'inhiber. D'autre part, Fak peut recruter Grb2-Sos directement, ou indirectement via Src, pour mener à l'activation de la petite GTPase Ras et ainsi stimuler la voie Raf-MEK/Erk. Cette dernière voie peut également être stimulée par certaines sous-unités $\alpha$ partenaires de $\beta 1$, via le recrutement/activation de Fyn ou de Yes, deux autres membres de la famille Src, et leur recrutement subséquent de Shc-Grb2-Sos. Raf phosphoryle l'homologue anti-apoptotique Bcl-2 pour le stabiliser, tandis que RSK-1, un effecteur de la voie MEK/Erk, phosphoryle Bad pour l'inhiber. Dans tous les cas, la stimulation des voies PI3-K/Akt et MEK/Erk produit généralement une régulation d'expression d'homologues Bcl-2 positive pour les anti-apoptotiques et négative pour les pro-apoptotiques, ainsi qu'une régulation positive d'expression d'IAP. Alternativement, Fak contribuera au recrutement de la paxilline, de la taline, de la vinculine et de Src pour former les points focaux d'adhérence, en association directe avec les microfilaments d'actine. Cette stabilisation du cytosquelette permet la séquestration des homologues pro-apoptotiques Bmf et Bim. À noter que d'autres voies de signalisation de survie stimulées par les intégrines $\beta 1-F a k$ (p130 ${ }^{\mathrm{CAS}}-\mathrm{Nck}-\mathrm{PAK}, \mathrm{PKC}, \mathrm{cFLIP}, \mathrm{etc}$.) existent, qui ne sont pas illustrées ici par souci de clarté et de concision. ASK-1 : apoptosis signal-regulating kinase-1; Erk1/Erk2 : extracellular regulated kinases 1 et 2 ; Fak : focal adhesion kinase; IAP : inhibitors of apoptosis proteases; ILK: integrin-linked kinase; IRS: insulin receptor substrate; MEK : mitogen-activated protein (MAP) kinase kinase; Pax : paxilline; PDK : PI3-K dependent kinase; PI3-K : phosphatidylinositol-3kinase; pro-CASP-9 : précurseur inactif de la caspase initiatrice CASP-9; Tal : taline; Vin : vinculine. 


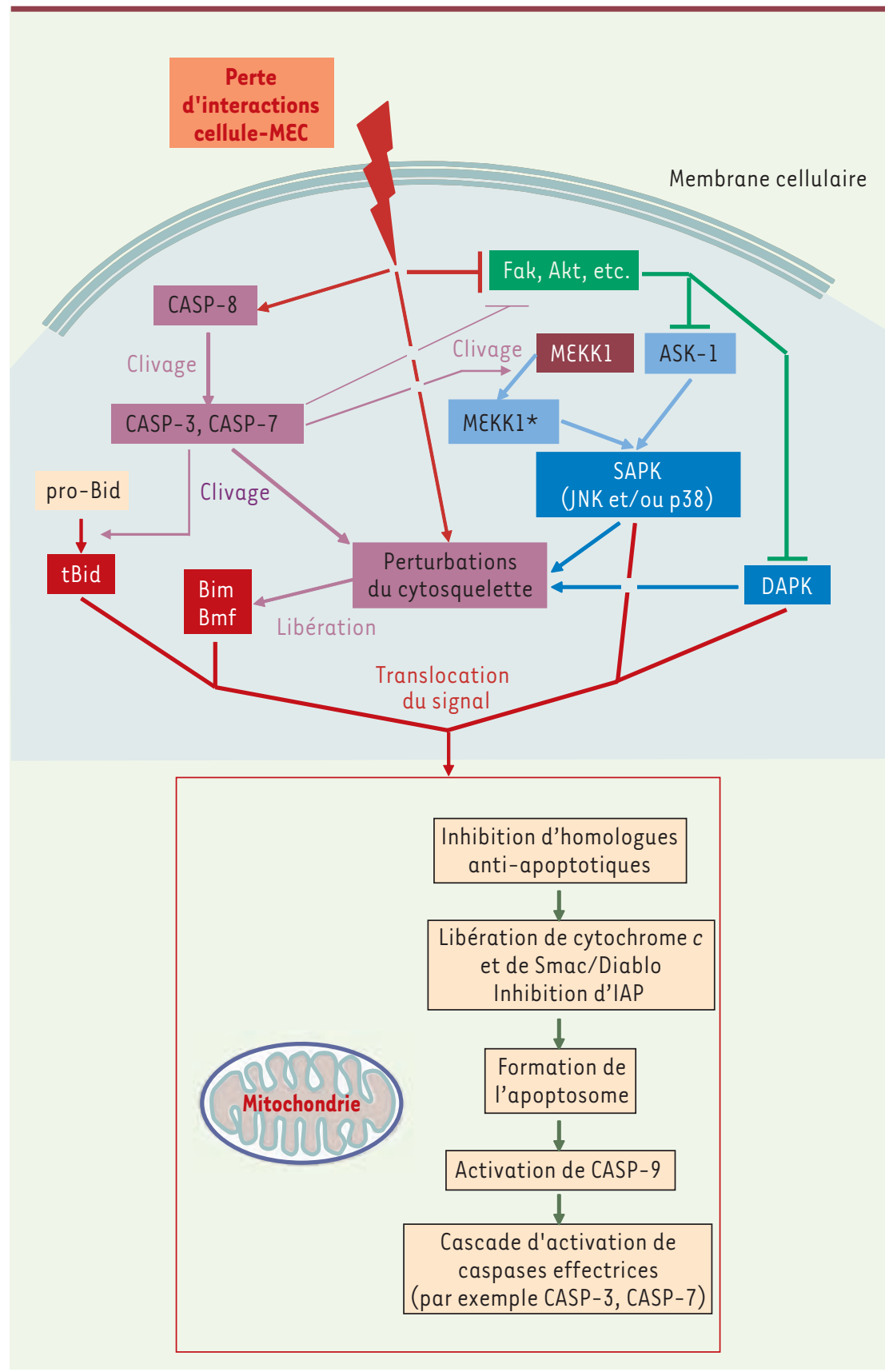

tent encore à être pleinement élucidés. Parmi les voies de transduction de signal stimulées par les intégrines, celles de la $\mathrm{PI3-K}$ et de MEK/Erk sont bien connues pour leurs rôles dans la survie cellulaire $[2,3$, 10-12, 15-18] (Figure 2). En ce qui concerne $\mathrm{PI3}-\mathrm{K}$, son activation mène normalement à l'activation d'Akt qui phosphoryle, notamemnt, les homologues pro-apoptotiques Bax et Bad, pour les inactiver, ainsi que le précurseur de la caspase initiatrice CASP-9, pour renforcer son état d'inactivation [2, 3 , 11, 12, 15-17] (Figure 2). Dans tous les cas, la stimulation des voies PI3-K/Akt ou MEK/ Erk aboutit généralement à une régulation d'expression d'homologues $\mathrm{Bcl}-2$ positive pour les anti-apoptotiques et négative pour les pro-apoptotiques, ainsi qu'à une régulation positive d'expression d'IAP $[2,3$, 11, 12, 15-23] (Figure 2). De ces faits, il a été démontré que l'inhibition de Fak induit fortement l'anoïkose, tandis que sa surexpression inhibe le processus $[3,10-12,14$, 15]. Cette importance de Fak dans la suppression de l'anoïkose serait liée au rôle de pivot qu'elle semble exercer dans la stimulation des voies PI3-K/Akt ou MEK/Erk par les intégrines, selon la composition de la cassette de signalisation qu'elle contribue à former [3, 7, 14, 15] (Figure 2). De plus, il faut noter que la complexité de signalisation de survie par les intégrines, toujours selon l'intégrine et le type cellulaire étudié, s'accroît en proportion de l'intervention sélective d'isoformes ou de membres familiaux distincts de kinases [16-19, 22].

Bien que la compréhension des voies de signalisation en jeu dans la suppression de l'anoïkose continue de progresser, la

Figure 3. Voie commune d'induction de l'anoïkose. La perturbation des interactions cellule-matrice extracellulaire (MEC) assurées par les intégrines a pour effet d'activer la caspase initiatrice CASP-8 qui, à son tour, active de manière dite «à faible intensité » des caspases effectrices comme CASP-3 et CASP-7. Ces dernières activent l'homologue Bcl-2 sensibilisateur (pro-apoptotique) Bid, et inactivent des kinases, comme Fak et Akt, associées à la signalisation par les intégrines. Elles clivent également MEKK1, un activateur en amont de certaines SAPK, comme les JNK, le rendant constitutivement actif («MEKKl*»). En parallèle, la perte de signalisation intégrines-Fak facilite l'activation de DAPK et ne supprime plus l'activation d'ASK-1, permettant son activation de certaines SAPK comme les JNK et p38. Par ailleurs, les perturbations du cytosquelette qui résultent de la perte d'interactions cellule-MEC, de l'action protéolytique des caspases effectrices, ainsi que de l'action des SAPK et DAPK activées, libèrent les homologues sensibilisateurs Bim et Bmf. Enfin, il intervient une translocation du signal vers les mitochondries par Bim, Bmf et tBid, ainsi que des SAPK et, on peut le présumer, des DAPK, menant à la formation ultime de l'apoptosome, à l'activation de CASP-9 et à la cascade d'activation de caspases effectrices (voir aussi Figure 1). ASK-1 : apoptosis signal-regulating kinase-1; DAPK : death associated protein kinases; Fak: focal adhesion kinase; JNK : c-Jun N-terminal kinases; MEKK1 : MEK (mitogen-activated protein (MAP) kinase kinase) kinase 1 ; SAPK : stress-activated MAP kinases. 
signalisation responsable de l'induction de l'anoïkose reste mal comprise [3, 10-12]. On sait que l'inhibition des interactions cellule-MEC se traduit non seulement par une perte de signaux de survie, causant ainsi une baisse d'activation de molécules/voies de signalisation comme Fak, PI3-K/Akt ou MEK/Erk, mais également par l'activation rapide de la caspase initiatrice CASP-8 [3, 10-12] (Figure 3). À son tour, l'activation de

CASP- 8 mène, entre autres, à l'activation de «faible intensité » de caspases

Entérocyte indifférencié

- Homologues $\mathrm{Bcl}-2$ prédominants : Anti-apoptotiques: $\mathrm{Bcl}-2, \mathrm{Bcl}-\mathrm{X}_{\perp}$ Pro-apoptotiques : Bax - p38 , Akt-3 : non exprimées

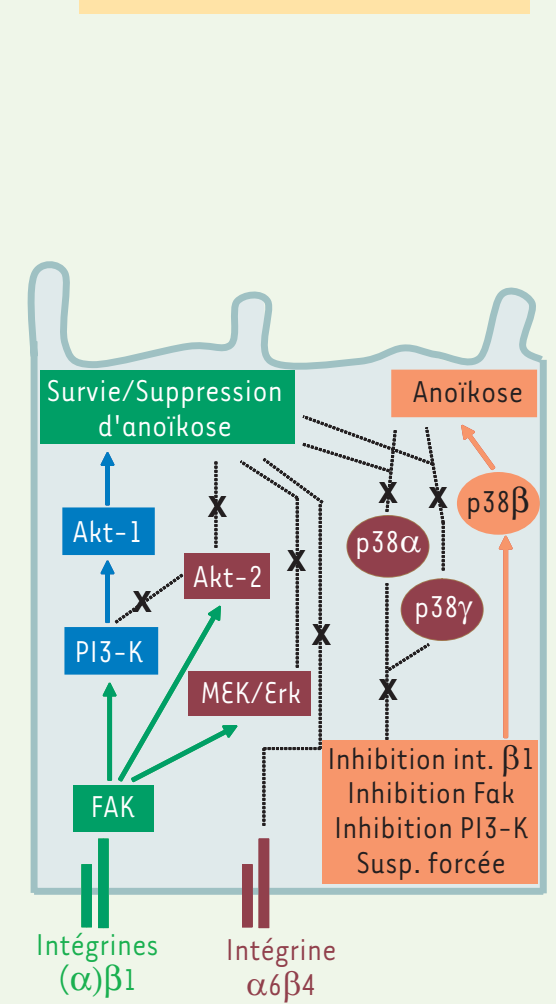

$\longrightarrow$ Influence/rôle

\section{Entérocyte différencié}

- Homologues $\mathrm{Bcl}-2$ prédominants : Anti-apoptotiques : $\mathrm{Bcl}-\mathrm{X}_{\mathrm{L}}, \mathrm{Mcl}-\mathrm{l}$

Pro-apoptotiques: Bax, Bak, Bad - p38 $\beta$, Akt-3 : non exprimées

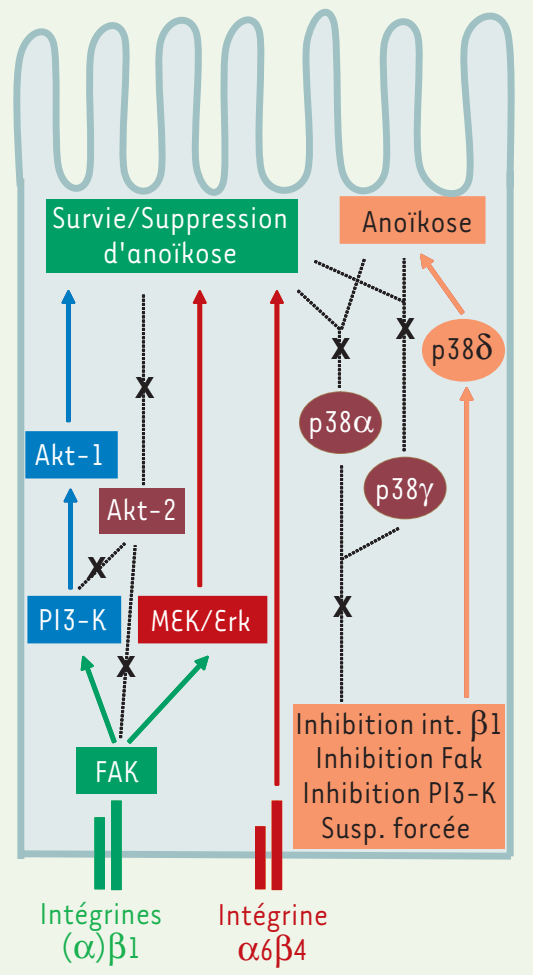
effectrices, au clivage de l'homologue pro-apoptotique Bid pour l'activer (tBid) et à la désactivation, par clivage, de kinases telles que Fak et Akt [3, 10-12] (Figure 3). De plus, les perturbations ressenties dans le cytosquelette par la perte d'adhésion ont pour effet de libérer les sensibilisateurs pro-apoptotiques Bim et $B m f$, qui sont normalement séquestrés dans les microtubules et les filaments d'actine, respectivement $[2,3,10-12]$ (Figures 2 et 3 ). Le tout conduit à l'activation de CASP-9 (Figure 3). L'anoïkose demeure donc un processus réversible tant que le signal d'induction ne s'est pas translocalisé vers les mitochondries pour mener à la formation de l'apoptosome [3, 10-12].

Le rôle des SAPK JNK et p38 dans la survie cellulaire est encore ambigu [3, 10-12, 18]. Ces kinases peuvent jouer un rôle de promotion de la survie ou d'induction d'apoptose/anoïkose, ou même ne jouer aucun rôle dans ces processus en fonction du type cellulaire et de la nature de l'isoforme étudiée [3, 10-12, 18, 22-25]. En ce qui concerne $\mathrm{p} 38$, la stimulation d'une isoforme engagée dans l'induction de l'anoïkose résulte généralement de la perte des signaux de survie conférés par

Figure 4. Mécanismes spécifiques de la survie entérocytaire selon l'état de différenciation. Les profils d'expression des homologues Bcl-2 sont distincts selon l'état de différenciation entérocytaire. Les interactions cellule-matrice extracellulaire (MEC) et les voies de signalisation exercent des rôles également distincts dans la survie cellulaire ou dans l'induction de l'apoptose/anoïkose (tel qu'illustré), ainsi que dans la modulation de l'expression des homologues Bcl-2 (non illustré), toujours selon l'état de différenciation. Dans les entérocytes indifférenciés (à gauche), les intégrines $\beta 1$, Fak, PI3-K et Akt-1 sont cruciales pour la survie et la suppression de l'anoïkose. Cependant, Akt-2 et la voie MEK/Erk ne jouent pas de rôle dans ces processus, bien que la signalisation intégrines $\beta 1 /$ Fak contribue à leur stimulation. Akt-2 est indépendante de PI3-K, tandis

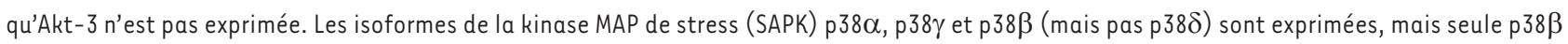
est requise pour l'induction de l'anoïkose. Enfin, l'intégrine $\alpha 6 \beta 4$ ne contribue pas au maintien de la survie. Dans les entérocytes différenciés (à droite), les intégrines $\beta 1$, Fak, PI3-K et Akt-l demeurent cruciales pour la survie, tandis que c'est p38 $\delta$ (et non p38 $\beta$, qui n'est plus exprimée) qui doit maintenant être requise pour l'induction de l'anoïkose. $p 38 \alpha$ et $p 38 \gamma$ (mais pas p38 $\beta$ ) sont encore exprimées, mais ne jouent toujours pas de rôle dans la survie ou l'induction de l'anoïkose. La voie MEK/Erk demeure influençable par la signalisation intégrines $\beta 1 / F a k$ et, ici, contribue à la survie. De plus, l'intégrine $\alpha 6 \beta 4$ contribue maintenant à la suppression de l'anoïkose. D'un autre côté, Akt-2 demeure indépendante de PI3-K, tout en étant maintenant indépendante de la signalisation intégrines $\beta 1 / F a k$; elle ne joue toujours pas de rôle dans la survie ou dans l'induction de l'apoptose/anoïkose. Enfin, Akt-3 n'est toujours pas exprimée. Fak : focal adhesion kinase; Erk : extracellular regulated kinase ; MEK : mitogenactivated protein (MAP) kinase kinase; PI3-K : phosphatidylinositol-3-kinase; SAPK : stress-activated MAP kinases. 
les interactions cellule-MEC, ainsi que de l'activation subséquente de kinases intervenant en amont, comme ASK-1 (apoptosis signal-regulating kinase-1) [3, 10, 18] (Figure 3). D'ailleurs, Akt est bien connue pour phosphoryler cette kinase, et ainsi l'inhiber [3, 11, 12, 16, 17] (Figures 2 et 3). Dans tous les cas, l'activité d'une SAPK lors de l'induction de l'anoïkose semble requise pour le blebbing membranaire, la déstabilisation du cytosquelette, la dégradation de l'ADN ou l'activation d'effecteurs apoptotiques (comme les caspases) [3, 10-12, 18, 22-25]. Dans le même temps, une SAPK peut contribuer à augmenter l'expression d'homologues pro-apoptotiques tout en diminuant celle d'anti-apoptotiques, en plus d'inhiber par phosphorylation certains anti-apoptotiques comme Bcl-2 [2, 3, 10-12, 18, 22, 23]. Enfin, d'autres kinases, comme les DAPK (death associated protein kinase), ont été identifiées au cours des dernières années, qui entrent également en jeu dans l'induction de l'apoptose et de l'anoïkose [3, 26] (Figure 3).

Dans leur ensemble, ces données révèlent que la régulation de la survie cellulaire, de l'apoptose ou de l'anoïkose constitue une affaire fort complexe, qui requiert d'autant plus des distinctions supplémentaires selon les tissus et les types cellulaires spécifiquement concernés. Or, des études récentes de la survie cellulaire effectuées dans un tissu particulier, l'épithélium intestinal humain, montrent qu'il existe un palier additionnel de complexité dans la régulation de ces différents processus.

\section{Survie cellulaire : régulation distincte selon l'état de différenciation}

Le renouvellement continu de l'épithélium intestinal s'effectue le long d'une unité bien définie, l'axe crypte-villosité. Cette unité est essentiellement constituée de deux populations cellulaires: les cellules prolifératrices et immatures de la crypte, et les cellules différenciées de la villosité [27]. Les entérocytes différenciés entrent en anoïkose lorsqu'ils atteignent l'apex des villosités, en guise d'exfoliation [27]. De leur côté, les cellules de la crypte peuvent entrer «spontanément» en apoptose, un processus, rare dans les conditions physiologiques normales, qui sert à évacuer les cellules filles endommagées ou défectueuses [27]. Cette apparente dualité de destinée entre les entérocytes indifférenciés et différenciés, couplée à des patrons différentiels d'expression d'homologues $\mathrm{Bcl}-2$ le long de l'axe crypte-villosité [27-29], suggère l'existence d'une régulation distincte de la survie entérocytaire selon l'état de différenciation [27]. De fait, le profil distinct d'expression d'homologues $\mathrm{Bcl}-2$ dans les entérocytes humains différenciés s'établit graduellement au cours du processus de différenciation [21] (Figure 4), et l'expression individuelle de ces mêmes homologues est sujette à une régulation distincte selon l'état de différenciation [21, 22]. L'insuline, notamment, fait chuter l'expression de Bax et Bak dans les entérocytes indifférenciés, tandis qu'elle fait plutôt chuter celle de Bad dans les entérocytes différenciés [21]. De surcroît, les voies PI3-K/Akt et MEK/Erk sont impliquées distinctement dans la suppression de l'apoptose, ainsi que dans la modulation de l'expression d'homologues $\mathrm{Bcl}-2$, toujours selon l'état de différenciation [20-22]. La voie MEK/Erk ne joue ainsi aucun rôle dans la survie des entérocytes humains indifférenciés, tandis qu'elle intervient dans la survie des entérocytes humains différenciés [20-22] (Figure 4).
Puisque la survie et l'apoptose entérocytaires sont bel et bien sujettes à des mécanismes spécifiques de régulation selon l'état de différenciation, en est-il de même pour la régulation de l'anoïkose? D'une part, les entérocytes différenciés ont une moindre susceptibilité face à l'anoïkose que les entérocytes indifférenciés [21, 22]. D'autre part, Fak, ainsi que les intégrines $(\alpha) \beta 1$ et $\alpha 6 \beta 4$, jouent des rôles distincts dans la suppression de l'anoïkose et dans la modulation de l'expression des homologues $\mathrm{Bcl}-2$, selon l'état de différenciation [21, 22]. Par exemple, l'intégrine $\alpha 6 \beta 4$ ne joue aucun rôle dans la survie des entérocytes indifférenciés, mais intervient dans la survie des entérocytes différenciés [21] (Figure 4). De plus, les isoformes d'Akt sont régulées et engagées sélectivement dans la suppression de l'anoïkose selon l'état de différenciation [19, 22] (Figure 4). Enfin, les isoformes de p38 sont engagées sélectivement dans l'induction de l'anoïkose, toujours selon l'état de différenciation [22, 25] (Figure 4). Par conséquent, la régulation de l'anoïkose entérocytaire humaine est également assujettie à des mécanismes spécifiques selon l'état de différenciation cellulaire (Figure 4). Cela s'accorde très bien avec le fait que les entérocytes expriment des profils distincts d'intégrines en fonction de leur état de différenciation et, de plus, interagissent avec des constituants de la $M E C$ spécifiques le long de l'axe crypte-villosité [5, 27].

\section{Conclusions}

Le concept d'une régulation distincte de la survie cellulaire, de l'apoptose et de l'anoïkose selon l'état de différenciation est nouveau. II va de soi que notre compréhension des mécanismes sous-jacents à la suppression et à l'induction «différenciation-spécifique » de l'apoptose et de l'anoïkose n'en est qu'à ses débuts. Or, il est intéressant de constater que ce nouveau concept s'applique à des types cellulaires autres que les entérocytes, et notamment aux cellules myogéniques $[23,24,30,31]$.

À l'instar de l'apoptose, il existe un nombre croissant de désordres pathologiques qui sont caractérisés par une dérégulation de l'anoïkose $[2,3,6,10-12,15,26,30$ 32]. Par ailleurs, le besoin de comprendre la régulation de la survie cellulaire, de l'apoptose et de l'anoïkose a récemment grandi en importance dans la recherche sur le cancer, d'autant que l'acquisition d'une résistance à l'anoïkose est maintenant perçue comme une étape critique dans la progression tumorale $[2,3,10-12,15$, 26, 32-34]. Un défi fondamental de taille qui se présente maintenant consiste donc à chercher les réponses à trois vastes interrogations: le «pourquoi » de l'existence de mécanismes distincts de régulation de l'apoptose et de l'anoïkose, selon l'état de différenciation cellulaire; le 
« comment» de ces mécanismes distincts, c'est-à-dire l'identification fonctionnelle de ligands extracellulaires, d'intégrines et de molécules/ voies de signalisation en cause, ainsi que leurs rôles «différenciationspécifiques » exercés dans la suppression de l'apoptose/anoïkose; enfin, le «en quoi » ces mêmes mécanismes «différenciation-spécifiques » contribuent à l'émergence et à l'établissement de pathologies particulières lorsqu'ils se trouvent dérégulés. Une meilleure compréhension des déterminants moléculaires de la survie cellulaire, de l'apoptose et de l'anoïkose, incluant toute distinction selon le type cellulaire et leur état de différenciation, devrait donc permettre de circonscrire davantage les normes physiologiques et leurs écarts physiopathologiques. $\diamond$

\section{SUMMARY}

\section{Cell survival: differences and differentiation}

The regulatory mechanisms of cell survival and apoptosis are very complex in nature, implicating numerous players and signaling pathways not only in the decision-making process of surviving (or dying), but as well as in the execution of apoptosis itself. The same complex nature applies with regards to anoikis, a form of apoptosis that is largely regulated by integrin-mediated, cell-extracellular matrix interactions. However, cell survival, apoptosis and anoikis also happen to implicate further mechanistic distinctions according to the specific tissue and/or cell type concerned. Incidentally, recent studies in a particular tissue, the human intestinal epithelium, have unearthed yet another layer of complexity in the regulation of these three cellular processes, namely the implication of differentiation state-specific mechanisms. Although our understanding of the molecular underpinnings of this new concept of differentiation state-distinct regulation of cell survival, apoptosis and/or anoikis is in its infancy, there is already evidence that such principle applies as well to cell types other than intestinal epithelial cells. Further studies on the differentiation state-specific regulation of these three cellular processes, either under normal or physiopathological situations, should prove crucial in increasing our understanding of pathologies which implicate a dysregulation of apoptosis and/or anoikis - such as cancer. $\diamond$

\section{RÉFÉRENCES}

1. Edinger AL, Thompson CB. Death by design : apoptosis, necrosis and autophagy. Curr Opin Cell Biol $2004 ; 16: 663-9$.

2. Cory S, Huang DCS, Adams JM. The Bcl-2 family : roles in cell survival and oncogenesis. Oncogene $2003 ; 22: 8590-7$.

3. Martin SS, Vuori K. Regulation of $\mathrm{Bcl}-2$ proteins during anoikis and amorphosis. Biochim Biophys Acta $2004 ; 1692$ : 145-7.

4. Fuentes-Prior $P$, Salvesen $G$. The protein structures that shape caspase activity, specificity, activation and inhibition. Biochem J 2004, 384 : 201-22.

5. Beaulieu JF. Integrins and human intestinal cell functions. Front Biosci $1999 ; 4: 310-21$.

6. Boudreau NJ, Jones PL. Extracellular matrix and integrin signaling : the shape of things to come. Biochem J $1999 ; 339: 481-8$.

7. Giancotti FG, Ruoslahti $\varepsilon$. Integrin signaling. Science $1999 ; 285: 1028-32$.

8. Giancotti FG. Complexity and specificity of integrin signaling. Nat Cell Biol 1999; 2 : ع13-4.

9. Hynes RO. Integrins : bidirectional, allosteric signaling machines. Cell 2002, $110: 673-87$.

10. Frisch SM, Screaton RA. Anoikis mechanisms. Curr Opin Cell Biol $2001 ; 13: 555-62$.

11. Grossmann J. Molecular mechanisms of « detachment-induced apoptosis-anoikis ». Apoptosis $2002 ; 7: 247-260$.

12. Stupack DG, Cheresh DA. Get a ligand, get a life : integrins, signaling and cell survival. J Cell Sci $2002 ; 115: 3729-38$.
13. Ameisen JC. Anoïkis. Med Sci (Paris) $2005 ; 21: 117$.

14. Cornillion J, Campos L, Guyotat D. Focal adhesion kinase (FAK), une protéine aux fonctions multiples. Med Sci (Paris) 2003 ; $19: 743-52$.

15. Giancotti FG. Integrin signaling : specificity and control of cell survival and cell cycle progression. Curr Opin Cell Biol $1997 ; 9: 691-700$.

16. Kim D, Dan HC, Park S, et al. AKT/PKB signaling mechanisms in cancer and chemoresistance. Front Biosci 2005, $10: 975-87$.

17. Song $G$, Ouyang $G, B a o S$. The activation of Akt/PKB signaling pathway and cell survival. J Cell Mol Med $2005 ; 9$ : 59-71.

18. Kyriakis JM, Avruch J. Mammalian mitogen-activated protein kinase signal transduction pathways activated by stress and inflammation. Physiol Rev 2001 ; 81 : 807-69.

19. Dufour G., Demers MJ, Gagné D, et al. Human intestinal epithelial cell survival and anoikis: differentiation state-distinct regulation and roles of protein kinase B/Akt isoforms. J Biol Chem 2004 ; 279 : 44113-22.

20. Gauthier R, Laprise P, Cardin $\varepsilon$, et al. Differential sensitivity to apoptosis between the human small and large intestinal mucosae : linkage with segment-specific regulation of $\mathrm{Bcl}-2$ homologs and involvement of signaling pathways. J Cell Biochem $2001 ; 82: 339-55$.

21. Gauthier R, Harnois C, Drolet JF, et al. Human intestinal epithelial cell survival : differentiation state-specific control mechanisms. Am J Physiol $2001 ; 280:$ C1540-54.

22. Harnois C, Demers M-J, Bouchard V, et al. Human intestinal epithelial crypt cell survival and death : complex modulations of $\mathrm{Bcl}-2$ homologs by Fak, PI3-K/Akt-1, MEK/Erk, and p38 signaling pathways. J Cell Physiol $2004 ; 198: 209-22$.

23. Laprise P, Vallée K, Demers MJ, et al. Merosin (Iaminin-2/4)-driven survival signaling : complex modulations of $\mathrm{Bcl}-2$ homologs. J Cell Biochem 2003; 89 : 1115-25.

24. Laprise P, Poirier દ̀M, Vézina $A$, et al. Merosin-integrin promotion of skeletal myofiber cell survival : differentiation state-distinct involvement of $p 60^{\mathrm{Fyn}}$ tyrosine kinase and $p 38 \alpha$ stress-activated MAP kinase. J Cell Physiol $2002 ; 191: 69-81$.

25. Vachon PH, Harnois C, Grenier A, et al. Differentiation state-selective roles of $\mathrm{p} 38$ isoforms in human intestinal epithelial cell anoikis. Gastroenterology 2002 ; $123: 1980-91$.

26. Shohat G, Shani G, Eisenstein M, Kimchi A. The DAP-kinase family of proteins: study of a novel group of calcium-regulated death-promoting kinases. Biochim Biophys Acta 2002 ; 1600 : 45-50.

27. Ménard D, Beaulieu JF, Boudreau F, Perreault N, Rivard N, Vachon PH. Gastrointestinal tract (GI tract). In : Unsicker K, Krieglstein K, eds. Cell signaling and growth factors in development. Verlag: Wiley-VCH, $2005: 755-90$.

28. Vachon PH, Cardin É, Harnois C, et al. Early establishment of epithelial apoptosis in the developing human small intestine. Int J Dev Biol 2000; $44: 891-8$.

29. Vachon $\mathrm{PH}$, Cardin $\varepsilon$, Harnois $C$, et al. Early acquisition of bowel segment-specific $\mathrm{Bcl}-2$ expression profiles during the development of the human ileum and colon. Histol Histopathol 2001 ; 16 : 497-510.

30. Vachon PH, Loechel F, Xu H, et al. Merosin and laminin in myogenesis: specific requirement for merosin in myotube stability and survival. J Cell Biol 1996; 134 : 1483-97.

31. Vachon $\mathrm{PH}, \mathrm{Xu} \mathrm{H}$, Liu L, et al. Integrins $(\alpha 7 \beta 1)$ in muscle function and survival : disrupted expression in merosin-deficient congenital muscular dystrophy. J Clin Invest1997; 100 : 1870-81.

32. Danen $\varepsilon H$, Sonnenberg A. Integrins in regulation of tissue development and function. J Pathol $2003 ; 201: 632-41$.

33. Kroemer G. Cell death and cancer : an introduction. Oncogene 2004 ; $23: 2744-5$.

34. Liotta AL, Kohn E. Cancer and the homeless cell. Nature 2004 ; $430: 973-4$.
TIRÉS À PART

P.H. Vachon 